\title{
Evaluación microbiológica en programas de alimentación escolar en instituciones educativas en el Departamento de Boyacá - Colombia
}

\author{
Microbiological Evaluation in School Supply Programs in Educational \\ Institutions in Boyacá Department - Colombia \\ Sandra Suescún-Carrero ${ }^{1}$, Sandra Avila-Panche ${ }^{2}$
}

\section{Resumen}

Objetivo. Evaluar la calidad microbiológica en programas de alimentación escolar en instituciones educativas en el Departamento de Boyacá - Colombia en el lapso 2013- 2015. Métodos. Estudio descriptivo de superficies vivas e inertes en comedores de las Instituciones Educativas del Departamento de Boyacá, en 100 municipios en el año 2013, 115 en el año 2014 y 123 en el año 2015. Los parámetros evaluados fueron: microorganismos Mesófilos aerobios, Coliformes Totales, Coliformes fecales, Staphylococcus aureus (Estafilococco Coagulasa Positiva). Resultados. Se tomaron 200 muestras en el año 2013, aislándose 104 positivas en superficies vivas y 149 en superficies inertes; encontrándose Coliformes Totales en 59,6\% en superficies vivas y 44,3\% en superficies inertes. 230 muestras en el año 2014, 158 positivas para superficies vivas y 148 para inertes, con predomino de Coliformes Totales en superficies vivas $(48,1 \%)$ y Mesófilos en superficies inertes (49,3\%) y 526 muestras en el ańo 2015, 256 positivas para superficies vivas y 406 para inertes encontrándose en superficies vivas Coliformes Totales con 47,6\%, Staphylococo coagulasa positiva con 3,1\%y Mesófilos en superficies inertes 45,3\%. Conclusiones. La Vigilancia que realiza la Secretaria de Salud de Boyacá en las instituciones educativas muestra que los resultados obtenidos en los aislamientos de las superficies inertes y vivas exceden los valores mínimos permitidos, hallazgos que evidencian el incumplimiento de las buenas prácticas de manufactura, de los hábitos de los manipuladores y de las condiciones sanitarias de los comedores escolares.

Palabras claves: Seguridad alimentaria, Coliformes, salud escolar, contaminación de alimentos, microbiología de alimentos.

\section{Abstract}

Objective. To evaluate the microbiological quality in school feeding programs in educative institutions in the Department of Boyacá-Colombia in 2013-2015. Methods. Descriptive study of live and inert surfaces in dining rooms of the Educative Institutions of the Department of Boyacá, in 100 municipalities in 2013, 115 in 2014 and 123 in 2015. The parameters evaluated were: aerobic mesophilic microorganisms, total coliforms, faecal coliforms, Staphylococcus aureus (Staphylococcus Coagulase Positive). Results. 200 samples were taken in 2013, 104 positive on

\footnotetext{
1. Bacterióloga, Magister en Administración en Salud, Grupo de Investigación del Laboratorio de Salud Pública de Boyacá, Secretaria de Salud

de Boyacá, Tunja, Colombia.

2. Bacterióloga y Laboratorista clínica, Grupo de Investigación del Laboratorio de Salud Pública de Boyacá, Secretaria de Salud de Boyacá,

Tunja, Colombia 
alive surfaces and 149 on inert surfaces; total coliforms being found in $59.6 \%$ on alive surfaces, on inert surfaces $44.3 \%$. 230 samples in 2014,158 positive on alive surfaces and 148 for inert surfaces, with predominance of total coliforms on alive surfaces $48.1 \%$ and mesophiles on inert surfaces 49.3\% and 526 samples in 2015, 256 surface positive on alive and 406 for inert material. Total Coliforms were found with 47.6\%, Coagulase positive Staphylococcus with 3.1\% and Mesophiles in inert surfaces $45.3 \%$. Conclusions. The Surveillance, carried out by the Boyaca's Secretary of Health in educative institutions, shows that the results obtained in the insulation of inert and alive surfaces exceed the minimum values allowed, findings that show the non-compliance with good manufacturing practices, habits of the manipulators and the sanitary conditions of the school canteens.

Keywords: Food Supply, Coliforms, School Health, Food Contamination, Food Microbiology.

\section{Introducción}

Los programas de Alimentación Escolares (PAE's) han sido institucionalizados en Colombia desde 1941, bajo la responsabilidad del Ministerio de Educación Nacional (MEN), el cuál fija las pautas para la asignación de recursos destinados a la dotación y funcionamiento de restaurantes escolares (1).

En Jamaica, en 1995, se observaron efectos favorables de una iniciativa de desayuno escolar en el funcionamiento cognitivo de los niños con problemas de desnutrición que recibieron complemento nutricional como merienda (2). Adicionalmente, en Perú, en la evaluación del Programa de Desayunos Escolares, se constató una relación positiva entre el consumo del desayuno y el rendimiento escolar (Ministerio de Salud y PRISMA, 2001) (3). La "Red de Protección Social Nicaragua" (RPS), logró efectos significativos en la matrícula y en la promoción de grado, estos resultados fueron más representativos en niños de las familias más pobres (4).

En Colombia, de acuerdo con los análisis realizados por el MEN, entre los años 2002 y 2011, la tasa de deserción intra - anual, pasó del 8\% al 4,53\%. No obstante esta mejora, la deserción fue mayor en las zonas rurales de algunos departamentos y para algunos grupos poblacionales, particularmente los más vulnerables. Por ejemplo, en 2011, la tasa de deserción de Bogotá fue de 3,95\%, mientras que en algunos departamentos como Guainía y Vichada la cifra superó el 10\% (1).

Dentro de los lineamientos constituidos para la ejecución de los PAE, se establece la importancia de la calidad e inocuidad de los alimentos suministrados en estos, como parte de la seguridad alimentaria y de su contenido de aporte calórico nutricional en pro del desarrollo de los beneficiarios. Para la Organización Mundial de la Salud (OMS), la higiene alimentaria comprende todas las medidas necesarias para garantizar la inocuidad sanitaria de los alimentos, manteniendo a la vez el resto de cualidades que les son propias, con especial atención al contenido nutricional (5). Uno de los factores que en mayor medida afectan a la Salud Pública es la higiene de los alimentos, la cual se encuentra determinada por múltiples factores que van desde la obtención de los alimentos, transporte, almacenamiento, manipulación, elaboración y consumo de los mismos, para lo cual el análisis microbiológico puede informarnos acerca del resultado real de todo el proceso, ya que la presencia de determinados microorganismos en los alimentos es una medida de su calidad sanitaria y además un indicador de la mala manipulación (6).

La transmisión de enfermedades a través del consumo de alimentos es un fenómeno ya conocido; 
sin embargo, recientemente en todo el mundo se ha confirmado el aumento de la frecuencia de los cambios en las causas predominantes y en la epidemiología de las Enfermedades Transmitidas por Alimentos (ETAs), razón por la cual se debe dar a conocer las fuentes de contaminación y el o los microorganismos que con mayor frecuencia y porcentaje tienen importancia en el consumo de alimentos para la población beneficiaria del PAE, especialmente si estas enfermedades llegan a ser transmitidas por alimentos que se preparan en las Instituciones Educativas, y ocasionadas por diferentes factores como la falta de higiene del personal manipulador, el lavado inadecuado de manos (Superficies Vivas) y de superficies inerte Regulares: (Mesón de cocina) e Irregulares: (utensilios de cocina) (5).

Se ha estimado que las enfermedades de origen alimentario causan de 6 a 81 millones de enfermos y hasta 9.000 muertos cada año en los Estados Unidos de América. En Colombia el comportamiento de las ETAs durante el año 2013 fue de 4296 y en Boyacá de 89 casos, en 2014 se registró un total de 9730 casos incluyendo las presentadas en las Instituciones Educativas, de las cuales 44 fueron en el Departamento de Boyacá. Y en 2015 se presentaron 10362 casos de los cuales 72 fueron en Boyacá (7).

En un estudio realizado en México en el año 2012, se encontró en las superficies vivas (mano cocinera y mano auxiliar) un recuento de microorganismos Mesófilos aerobios y Coliformes totales que superó los límites permitidos. En las superficies inertes, la tabla de picar superó los límites permitidos, y en la barra de preparación de alimentos no superó los límites permitidos de Mesofilos y Coliformes totales (5). En otro estudio realizado en Argentina se reportó que el $43 \%$ de las muestras de las manos de los manipuladores fue positiva para Coliformes totales y el $21 \%$ para Staphilococus aureus, en las superficies inertes el $61 \%$ de los utensilios de cocina fue positivo para Coliformes totales (8). En el año 2007 se realizó un estudio en cinco ciudades de Colombia reportando que el $0,5 \%$ de los ma- nipuladores presentaba Staphilococus aureus en las manos (9) y en el año 2011 se publicó un estudio realizado en los puntos operativos del programa nacional de alimentación al adulto mayor reportando presencia de Staphilococus aureus en manos y uñas (10). El objetivo del presente estudio es determinar el riesgo microbiológico en programas de alimentación escolar (PAE) en instituciones educativas en el Departamento de Boyacá-Colombia en el lapso 2013- 2015.

\section{Materiales y métodos}

Se realizó un estudio descriptivo transversal de superficies vivas e inertes en los comedores de las Instituciones Educativas del Departamento de Boyacá, durante el periodo 2013 a 2015, en 100 municipios en el año 2013, 115 en el 2014 y 123 municipios para el año 2015; adquiriendo como base los listados de las Instituciones Educativas de dichos municipios, suministrados por la Secretaria de Educación del Departamento.

Los Técnicos de Saneamiento Ambiental del Departamento de Boyacá se capacitaron para la toma, transporte y conservación de las muestras de las superficies vivas e inertes, la toma de las muestras para superficies vivas se realizó en personas que tenían contacto directo con la manipulación de los alimentos a preparar y en las superficies inertes que tenían mayor contacto con los alimentos, tales como: mesones, tablas de picado y utensilios de cocina entre otros. Para la recolección de las muestras se empleó la técnica del hisopo, que consistió en frotar sobre un área determinada un hisopo estéril humedecido con la solución (medio de transporte: agua peptonada al $0,1 \%$ y/o solución salina al $0,85 \%$ ) para recoger la flora microbiana de dichas superficies, después se suspendió en un tubo de ensayo con 10 $\mathrm{ml}$ del medio de transporte para preservar la muestra en refrigeración. El transporte de las muestras se realizó bajo condiciones de refrigeración $\left(2-8^{\circ} \mathrm{C}\right)$ en neveras térmicas que garantizaron la cadena de frio hasta la entrega de la muestra al Laboratorio Departamental de Salud Pública de Boyacá (LDSP), 
donde se efectuó en el área de microbiología de alimentos el análisis de las muestras, mediante los métodos oficiales establecidos en cada caso (The International Commission on Microbiological Specifications for Foods (ICMSF) (11) y consistió en llevar a cabo el recuento en placa e identificación de aquellos microorganismos para los que se establecen límites en la Reglamentación Técnico-Sanitaria de superficies según la Normatividad: Norma Oficial Mexicana-093-SSA1-1993; $(12,13)$ y Resolución Ministerial No 363-2005-MINSA. PERÚ (14), estas normas tienen la finalidad de asegurar la calidad e inocuidad de los alimentos que se preparan en establecimientos fijos. Los parámetros evaluados fueron microorganismos mesófilos aerobios, Coliformes Totales, Coliformes de origen fecal, Staphylococcus aureus (Estafilococo Coagulasa Positiva).

\section{Resultados}

Se tomaron un total de 200 muestras en el año 2013, aislándose 104 muestras positivas para superficies vivas y 149 muestras positivas para superficies inertes, 230 muestras en el año 2014, aislándose 158 muestras positivas para superficies vivas y 148 positivas para superficies inertes y 526 muestras en el año 2015 obteniéndose 256 positivas para superficies vivas y 406 para superficies inertes.

Los resultados obtenidos en el año 2013 en los comedores de las instituciones educativas del Departamento de Boyacá reflejaron un predominio de Coliformes Totales tanto en superficies vivas como en superficies inertes (ver Tabla 1).

Tabla 1. Aislamiento en 200 muestras de superficies vivas e inertes de Instituciones Educativas del departamento de Boyacá en el año 2013.

\begin{tabular}{|l|r|r|r|r|}
\hline & Superficies vivas & \multicolumn{2}{|r|}{ Superficies inertes } \\
\hline Mesófilos & $\mathrm{N}$ & $\%$ & $\mathrm{~N}$ & $\%$ \\
\hline Coliformes Totales & 14 & 13,5 & 53 & 35,6 \\
\hline Coliformes Fecales & 62 & 59,6 & 66 & 44,3 \\
\hline Staphylococo coagulasa positiva & 28 & 26,9 & 30 & 20,1 \\
\hline Total & 0 & 0 & - & - \\
\hline
\end{tabular}

En la Tabla 2 se puede observar que en el año 2014 se presentó un predomino de Coliformes Totales en superficies vivas y de Mesófilos en superficies inertes.

Tabla 2. Aislamiento en 149 muestras de superficies vivas e inertes de Instituciones Educativas del departamento de Boyacá en el año 2014.

\begin{tabular}{|l|r|r|r|r|}
\hline Análisis Microbiológico & Superficies vivas & \multicolumn{2}{|r|}{ Superficies inertes } \\
\hline Mesófilos & $\mathrm{N}$ & $\%$ & $\mathrm{~N}$ & $\%$ \\
\hline Coliformes Totales & 70 & 44,3 & 73 & 49,3 \\
\hline Coliformes Fecales & 76 & 48,1 & 63 & 42,6 \\
\hline Staphylococo coagulasa positiva & 12 & 7,6 & 12 & 8,1 \\
\hline Total & 0 & 0 & - & - \\
\hline
\end{tabular}


Durante el año 2015 se presentó un predomino de Coliformes Totales, presencia de Staphylococo coagulasa positiva en superficies vivas y de mesófilos en superficies inertes (ver Tabla 3).

Tabla 3. Aislamiento en 526 muestras de superficies vivas e inertes de Instituciones Educativas del departamento de Boyacá en el año 2015.

\begin{tabular}{|l|r|r|r|r|}
\hline Análisis Microbiológico & Superficies vivas & \multicolumn{2}{|c|}{ Superficies inertes } \\
\hline Mesófilos & $\mathrm{N}$ & $\%$ & $\mathrm{~N}$ & $\%$ \\
\hline Coliformes Totales & 91 & 35,5 & 184 & 45,3 \\
\hline Coliformes Fecales & 122 & 47,6 & 163 & 40,1 \\
\hline Staphylococo coagulasa positiva & 35 & 13,7 & 59 & 14,6 \\
\hline Total & 8 & 3,1 & - & - \\
\hline
\end{tabular}

\section{Discusión}

En el presente estudio se encontró presencia de coliformes totales, coliformes fecales y mesófilos en las muestras estudiadas (tablas de picar, cuchillos, cucharas, mesones y manos de manipuladores de alimentos), quedando en evidencia la presencia de contaminación fecal y las inadecuadas prácticas de higiene y desinfección, datos que coinciden con lo reportado en un estudio realizado en una guardería en México, donde encontraron la presencia de coliformes fecales y mesofilos (15), así como tambien, en otros estudios realizados en Colombia (16).

Los resultados obtenidos en el análisis de las manos de los manipuladores son similares a los reportados en estudios realizados en Venezuela (17), Argentina (8) y México (5) reportando resultados que sobrepasan los límites establecidos de mesófilos, coliformes totales y coliformes fecales. La presencia de Staphylococo coagulasa positiva en este estudio fue de $3,1 \%$ coincidiendo con lo hallado en otros estudios, como en Argentina en el que se aisló Staphylococo coagulasa positiva en las manos del 21\% de manipuladores de áreas de producción de un supermercado (8), y en Colombia, donde se realizó un estudio en restaurantes de cinco ciudades encontrando $0,52 \%$ de individuos positivos (9). Los anteriores resultados indican la falta de lavado de manos después de usar los sanitarios y las deficientes prácticas higiénicas de los manipuladores, aumentando el riego de transmitir enfermedades gastrointestinales.
La Vigilancia de los factores de riesgo del ambiente y el consumo, que realiza el grupo de salud ambiental de la Secretaria de Salud de Boyacá en las instituciones educativas dentro del (PAE), muestra que los resultados obtenidos en los aislamientos de las superficies inertes y vivas exceden los valores mínimos permitidos, hallazgos que evidencian el incumplimiento de las buenas prácticas de manufactura, de los hábitos de los manipuladores y de las condiciones sanitarias de los comedores escolares. Se sugiere intensificar el plan de capacitación continuo y permanente al personal manipulador de alimentos de las instituciones educativas, desde el momento de su contratación y ser reforzados mediante charlas, cursos u otros medios efectivos de actualización, de acuerdo a lo establecido en la resolución 019 de 2013 (18-22).

\section{Agradecimientos}

A los Técnicos de Saneamiento Ambiental del Departamento de Boyacá, por la toma y transporte de las muestras.

\section{Referencias}

1. Ministerio de Educación. Lineamiento Técnico Administrativo del Programa de Alimentación Escolar (PAE). Página Oficial Ministerio de Educación Colombia. 2015.

2. 2. Chandler A, Walker S, Connolly K., Graham-MacGregor S. School breakfast improves verbal fluency in undernourished Jamaican children. The Journal of Nutrition 1995; 125: 894 -899 . 
3. Ministerio de Salud Del Perú. Evaluación de impacto del Programa de Desayunos Escolares sobre la educación y nutrición de los escolares: 2001

4. Caldés N, Coady D, Maluccio J. The cost of poverty alleviation transfer programs: A comparative analysis of three programs in Latin America. Food Consumption and Nutrition Division of International Food Policy Research Institute. 2004; paper No (174). Disponible en: http://www.eldis.org/ vfile/upload/1/document/0708/DOC22752.pdf

5. Organización Panamericana de la Salud - Organización Mundial de la Salud. Principios generales de Higiene de los alimentos del Codex. Washington; 2015 (citado 30 de julio 2017). Disponible en http:// www.paho.org/hq/index.php?option=com_content...higiene

6. Pérez-Silva M, Belmonte S, Martínez J. Estudio microbiológico de alimentos elaborados en comedores colectivos de alto riesgo. Rev Esp Salud Pública. 1998; 72: 67-75.

7. Instituto Nacional de Salud. Sivigila- vigilancia Rutinaria por Eventos Departamental. Bogotá: Instituto Nacional de Salud; 2014. Disponible en: http://www.ins.gov.co/lineas-de-acción/ subdirección-vigilancia/sivigila/paginas/vigilanci-rutinaria. aspx

8. Arzu O, Peiretti H, Rolla R, Roibon W. Evaluación de riesgo microbiológico en superficies inertes y vivas de manipuladores en áreas de producción de un supermercado del nordeste argentino. UNNE. 2000; 17:6-10.

9. Flórez A, Rincón C, Garzón P, Vargas N, Enríquez C. Factores relacionados con enfermedades transmitidas por alimentos en restaurantes de cinco ciudades de Colombia 2007. Asociación Colombiana de Infectología. 2008; 12:255-266.

10. Bejarano Roncancio J, Fandiño Martínez M. Caracterización de las condiciones higiénico sanitarias y microbiológicas de los Puntos Operaticos del Programa Nacional de Alimentación al Adulto Mayor PNAAM ICBF 2007. Revista de la Facultad de Medicina. 2011; 59:308-318

11. The Intemational Commission on Microbiological Specifications for Foods (ICMSF) update food control. 2006. Disponible en: http://www.icmsf.org/pdf/FSO\%20Ojectives/GuiaSimplificadoEnglish.pdf

12. Norma Oficial Mexicana-093-SSA1-1993. Prácticas de higiene y sanidad en la preparación de alimentos que se ofrecen en establecimientos fijos. 1993

13. Norma Oficial Mexicana-093-SSA1-1994. Prácticas de higiene y sanidad en la preparación de alimentos que se ofrecen en establecimientos fijos. 1994

14. Resolución Ministerial No 363-2005-MINSA.PERÚ. Norma sanitaria para el funcionamiento de restaurantes y servicios afines. 2005

15. Alonzo-Salomon J, Heredia-Navarrete M, Garcia-Roque O. Coliformes fecales y Mesofilos aerobios en alimentos superficies y manos del personal y niños de una guardería. Re Biomed. 2006; 17:86-95

16. Campuzano F Silvia, Mejía Flórez Dayana, Madero Ibarra
Catalina, Pabón Sánchez Paola. Determinación de la calidad microbiológica y sanitaria de alimentos preparados vendidos en la vía pública de la ciudad de Bogotá D.C. Nova [Internet]. 2015 Jan [citado 2017 may 14] ; 13(23): 81-92. Disponible en: http://www.scielo.org.co/scielo.php?script=sci_arttext\&pid=S1794-24702015000100008\&lng=en.

17. Lugo NV, Villalobos LB, Martínez R. Evaluación microbiológica en manipuladores de alimentos de tres comedores públicos en Cumana - Venezuela. Rev. Soc. Ven. Microbiol. 2006; 26: $95-100$

18. Ministerio de Salud y Protección Social. Resolución 2674 de 2013, por el cual se reglamenta el artículo 126 del Decreto Ley 019 de 2012 y se dictan otras disposiciones. Bogotá. 2013.

19. Ramírez, L. C. C., et al. Determinación de la presencia de bacterias patógenas para el humano en aguas de riego en la cuenca alta de la sabana de Bogotá; DC Colombia. Nova. 2014; 12(22).

20. Campuzano, S., et al. Determinación de la calidad microbiológica y sanitaria de alimentos preparados vendidos en la vía pública de la ciudad de Bogotá DC. 2015; Nova 13(23): 8192.

21. Flórez, R. A. N. Avances y perspectivas en Síndrome de Asperger. 2014; Nova, 12(21).

22. Rodríguez, O. E., Andrade, W. A., Díaz, F. E., \& Moncada, B. Actividad antimicrobiana de líquenes de la cuenca alta del rio Bogotá. 2015; Nova, 13(23). 